\title{
Archéopages
}

Archéopages

Archéologie et société

\section{Les conditions de détention pendant la deuxième guerre mondiale. Le camp de prisonniers de Stenay (Meuse)}

\section{Laurent Vermard et Frédéric Adam}

\section{(2) OpenEdition \\ Journals}

Édition électronique

URL : https://journals.openedition.org/archeopages/551

DOI : 10.4000/archeopages.551

ISSN : 2269-9872

Éditeur

INRAP - Institut national de recherches archéologiques préventives

Édition imprimée

Date de publication : 1 septembre 2014

Pagination : 70-75

ISSN : $1622-8545$

\section{Référence électronique}

Laurent Vermard et Frédéric Adam, «Les conditions de détention pendant la deuxième guerre mondiale. Le camp de prisonniers de Stenay (Meuse) ", Archéopages [En ligne], 39 | 10/2013-01/2014, mis en ligne le 01 janvier 2016, consulté le 21 janvier 2022. URL : http://journals.openedition.org/ archeopages/551; DOI : https://doi.org/10.4000/archeopages.551 


\section{Les conditions de détention pendant la deuxième guerre mondiale Le camp de prisonniers de Stenay (Meuse)}

Laurent Vermard Irrap

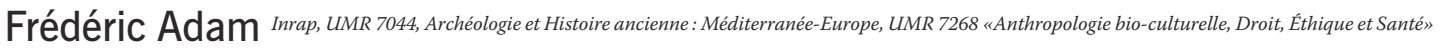
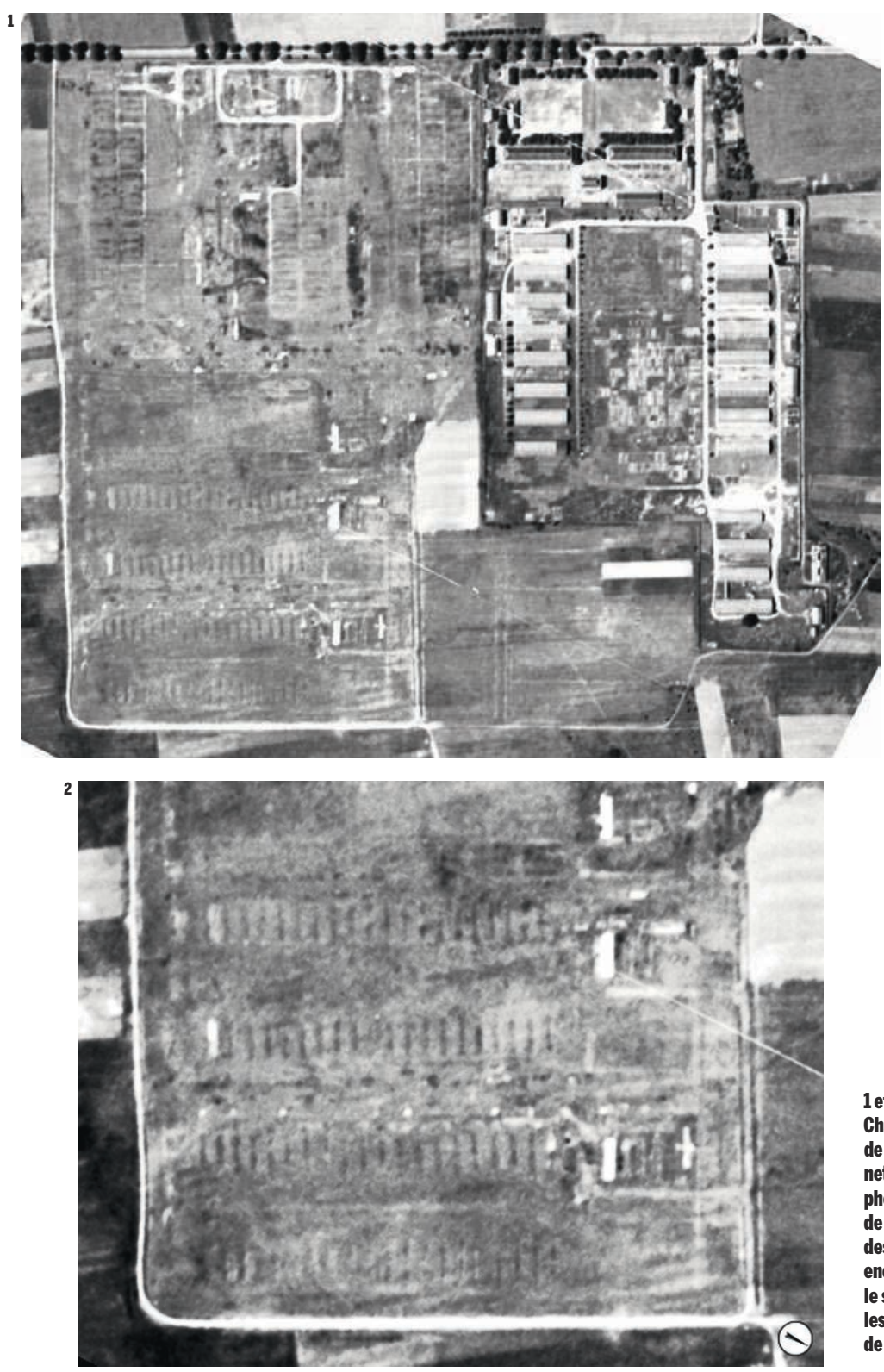
Au début de la deuxième guerre mondiale, la caserne Chanzy à Stenay, en Lorraine, qui abrite depuis 1935 le $155^{\mathrm{e}}$ Régiment d'Infanterie des forteresses, est occupée par les Allemands. Ils y installent un camp d'internement de soldats français, le $104{ }^{\mathrm{e}}$ CTE (Compagnie de Travailleurs Étrangers), qui fonctionne entre 1939 et juin 1940 (www.ajpn.org). En 1944, les Américains réoccupent la caserne Chanzy, dans laquelle ils aménagent, à partir du 6 novembre, un camp de transit, le CCPWE n ${ }^{\circ} 17$ (Continental Central Prisoners of War Enclosures). Ce dernier était prévu pour accueillir 10 ooo prisonniers allemands (Théofilakis, 2010, 2014) qui, arrivés à Stenay, étaient séparés des officiers et des nazis, avant d'être acheminés vers d'autres camps, ou bien de retourner à la vie civile.

\section{L'organisation du camp}

Le camp comprenait la caserne et tout le terrain au sud de ses murs. L'emprise est visible sur une photographie aérienne de 1950 observable sur le site internet Géoportail [ill. 1 et 2]. Cette photo montre des parcelles stigmatisées par la présence du camp de prisonniers avec notamment les limites claires de l'emplacement des chemins de desserte internes et les cantonnements comme gravés dans la terre végétale. Il semble divisé en trois parties. La première correspond aux bâtiments de casernement des militaires américains, le deuxième à la zone d'arrivage et de tri des prisonniers situés - d'après différents témoignages dont celui de Horst Kaiser, prisonnier allemand du camp de Stenay en 1946 (Kaiser, 1945-1946) - dans la zone d'entrepôts derrière les bâtiments. Le troisième, hors les murs, correspond au secteur de concentration des prisonniers maintenus en captivité par un enclos de fil barbelé. C'est dans cette dernière partie qu'ont été réalisés, en 2007 et 2013, deux diagnostics archéologiques ${ }^{\mathbf{1}}$ (Vermard, 2007 ; Vermard, 2013). Ces opérations ont permis d'appréhender la manière dont étaient traités les vaincus allemands à la fin de la Seconde Guerre mondiale.

Le cantonnement des prisonniers se faisait sous des tentes collectives installées à même un lit de crasse (résidus des hauts fourneaux de Stenay) - destiné à assainir le sol argileux - ce qui lui valu d'être nommé « le camp de pierres » par les prisonniers (Fusshöller, 1998 - prisonnier allemand du camp de Stenay en 1945). Sous ces abris de toile disposés en plusieurs rangées parallèles séparées par des allées, les soldats allemands dormaient à même le sol, ou parfois sur des couches de paille, protégés du froid par des couvertures (Mussbacher, 1945 - prisonnier allemand du camp de Stenay en 1945 ; Kaiser, 19451946). Ces différents éléments de couchage ont d'ailleurs été retrouvés dans les fosses d'aisance ainsi que de nombreux éléments de tentes (toile, fixations, tendeurs...) [ill. 3]. Les dimensions

observées sur la photo aérienne et le mobilier retrouvé sur le terrain correspondent à des tentes de type US « Hospital ward M-1941 » mesurant $15 \mathrm{~m} \times 5 \mathrm{~m}$ prévues pour 25 lits. Or, selon $\mathrm{H}$. Kaiser, la capacité des tentes a pu atteindre jusqu'à 134 personnes.

De nombreux dépotoirs et fosses d'aisance [ill. 4] ont été découverts dans l'une des allées centrales. Une dalle de sol en béton ne présentant pas d'amorce de mur sur son côté visible, mise au jour lors de l'opération de diagnostic de 2013 et repérable sur la photo de 1950, supportait probablement un bâtiment en tôle ou en bois. Ces vestiges et la présence de réseaux d'adduction d'eau (et de regards dans d'autres sondages) traduisent l'existence de bâtiments sanitaires.

En bordure nord-est de la zone de cantonnement, un petit carré de terrain ( $38 \mathrm{~m}$ de côté) délimite un espace plus protégé. Cette zone est entourée d'un fossé comblé, dans lequel a été déroulé du fil barbelé.

\section{La vie quotidienne au camp : alimentation, hygiène et économie de survie}

La majorité des artefacts exhumés concernent l'alimentation. Ont ainsi été retrouvés de nombreux contenants américains, tels que boîtes de conserve, sachets de produits lyophilisés - soupe, nescafé et jus de fruits (citron, orange, raisin) [ill. 5]. L'absence de vestiges de nourriture carnée dans les structures archéologiques échantillonnées s'accorde avec une enquête de l'armée américaine (Preventive medecine in world war II, 1969, p. 390) et avec les témoignages des prisonniers, qui évoquent une alimentation composée de rations des troupes américaines, ou parfois de simples bouillons clairs de légumes. La majorité des ustensiles de cuisine (couverts, plateaux repas, gamelles) découverts sur le terrain proviennent également de l'armée américaine, quelques récipients, gourdes et beurriers appartenant toutefois au paquetage de soldats allemands. De très rares éléments de vaisselle civile ont été mis au jour. Par ailleurs, certains objets découverts lors des fouilles suggèrent un détournement de leur fonction d'origine pour pallier le manque de récipients, par exemple des mines antipersonnel allemandes en verre utilisées comme bocaux [ill. 6].

Des sanitaires et des lieux de commodités étaient aménagés dans l'enceinte. Si la découverte de canalisations atteste la présence d'aménagements de points d'eau pour les prisonniers allemands, ils n'existaient apparemment pas en 1940 lorsque les combattants français vaincus furent eux-mêmes détenus sur le site (Dufilho, 2002, p. 194-199 prisonnier français en 1940 à Stenay). Les latrines consistaient en de simples aménagements installés sur d'étroites tranchées à usage collectif. Elles étaient régulièrement vidangées dans les champs alentour, comme le témoigne monsieur André Lorrain², habitant de Stenay, âgé de 14 ans à l'époque (Vermard, 2013). Ces feuillées ont aussi pu servir de dépotoir dans la dernière phase du camp : dans
"Aux Cailloux"

en 2007 ; responsab

d'opération : Lauren

réalisé aux « Groseilliers »

à Stenay en 2013 ;

responsable d'opération :

2. Témoignage ora

numérique intégré

au rapport d'opération

de diagnostic 


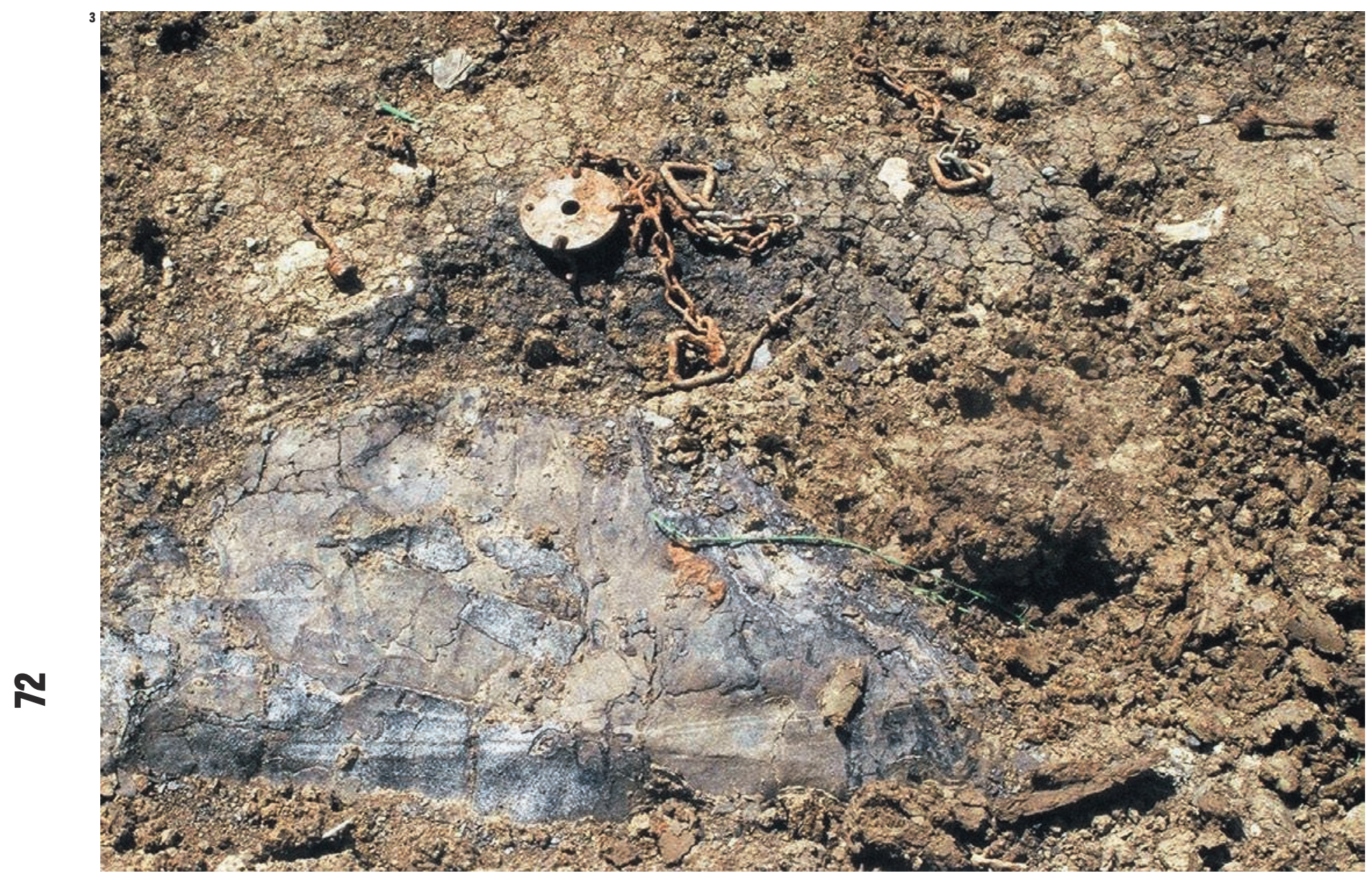

3. Au cours du diagnostic mené « Au Cailloux ॥), les

vestiges d'une tente ont

été mis au jour : on

aperçoit sur ce cliché la

toile, ainsi que les

chaines de fixation et des

tendeurs.

4. Des fosses ayant servi

de dépotoir ont permis la

découverte d'un

important matériel lié à

l'occupation du camp.

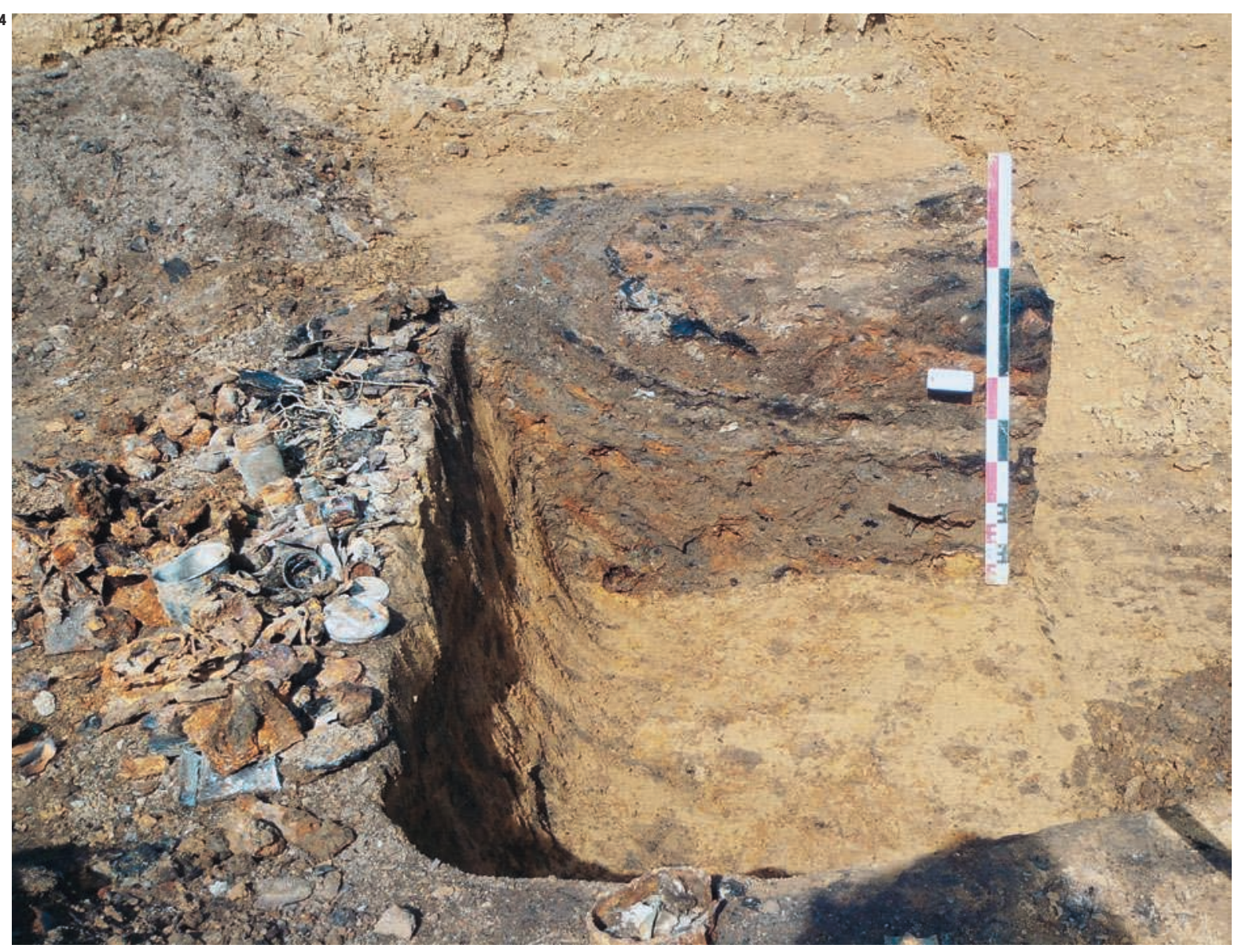


leur comblement ont été retrouvés divers objets liés à l'hygiène corporelle, savons, rasoirs et tubes de crèmes à raser, produits capillaires et peignes, pâtes dentifrices et brosses à dents [ill. 7]. Parmi ces objets, nous remarquons trois pains de savon RIF 0256. La présomption, portée par certains textes de propagande alliée, qu'ils aient été fabriqués par les nazis à partir de graisse humaine est aujourd'hui rejetée. Ces savons et une boîte de dentifrice de marque Rosodont sont les seuls artefacts d'origine germanique relatifs à l'hygiène. Le reste du matériel d'hygiène corporelle était donc fourni aux vaincus par l'armée américaine. Les conditions d'hygiène restaient cependant insuffisantes. La promiscuité aidant, puces et poux de corps ont trouvé un terrain de prolifération idéal. Pour lutter contre ce fléau, l'armée américaine semble avoir utilisé pour la première fois dans le camp de Stenay la pulvérisation au DDT (Fusshöller, 1998 ; Preventive medecine in world war II, 1969).

À la suite de la débâcle de l'armée allemande, les Américains se sont trouvés submergés devant l'afflux de prisonniers toujours plus nombreux. La conséquence en fut une concentration importante d'individus qui, affaiblis par les mauvaises conditions d'hygiène et l'insuffisance de nourriture, étaient en proie aux infections entériques de type dysenterie. Un rapport de l'armée américaine (Preventive medecine in world war II, 1969) mentionne qu'un quart des prisonniers des camps de transit étaient reconnus malades chaque semaine et que les morts étaient nombreux. F. J. Mussbacher (Mussbacher, 1945) rapporte également des décès à la suite de chutes, notamment nocturnes, dans les latrines. Le lieu d'inhumation de ces victimes est encore inconnu. Pourtant des soins étaient prodigués et des médicaments distribués comme l'attestent les divers contenants de produits pharmaceutiques découverts dans les dépotoirs (fioles, flacons, pots à onguents, boîtes d'aspirine) [ill. 8].
La mise au jour de très nombreux objets recyclés et transformés témoigne d'une économie de survie au sein de cet espace concentrationnaire. Bois, cuirs, caoutchouc, métaux, plastiques, etc., provenant d'objets divers, comme l'attestent notamment les nombreuses chutes découpées de chaussures et de pneus, ont servis à la confection de nouveaux objets afin d'améliorer un quotidien difficile. C'est notamment le cas de la fabrication de porte-manteaux à l'aide de fils de cuivre ou de chaussures. Trois modèles différents ont pu être identifiés. Au simple brodequin découpé et transformé en pantoufle, s'ajoute la création de nouvelles chaussures, comme l'élaboration de sabots et de sandalettes de type «Birkenstock» [ill. 9] à partir de matériaux hétéroclites.

Les recherches archéologiques réalisées dans le camp de transit de Stenay nous renseignent relativement bien sur les conditions de détention des prisonniers allemands. Les Américains semblent avoir essayé d'apporter les nécessités vitales, alimentaires et hygiéniques, à l'aide de leurs rations. Mais il faut relativiser ces apports et surtout noter l'absence de nourriture carnée. Des soins médicamenteux ont pu également être prodigués aux malades. Le dénuement des prisonniers n'en reste pas moins flagrant, qu'il s'agisse du couchage ou des nécessités vestimentaires.

Limplantation des cantonnements, l'aménagement de sanitaires et de commodités laissent transparaître une volonté d'organisation rationnelle de ce camp qui devait accueillir 10 ooo prisonniers. Toutefois, ces infrastructures se sont vite trouvées insuffisantes face à un afflux de prisonniers très supérieur aux estimations. 17 ooo prisonniers y sont dénombrés à la fin de la guerre (Théofilakis, 2010, 2014). Les conséquences de cette surpopulation sont bien connues à travers la littérature, toutes époques confondues, et ont entraîné une forte dégradation des conditions de détention tant au niveau alimentaire que sanitaire. 

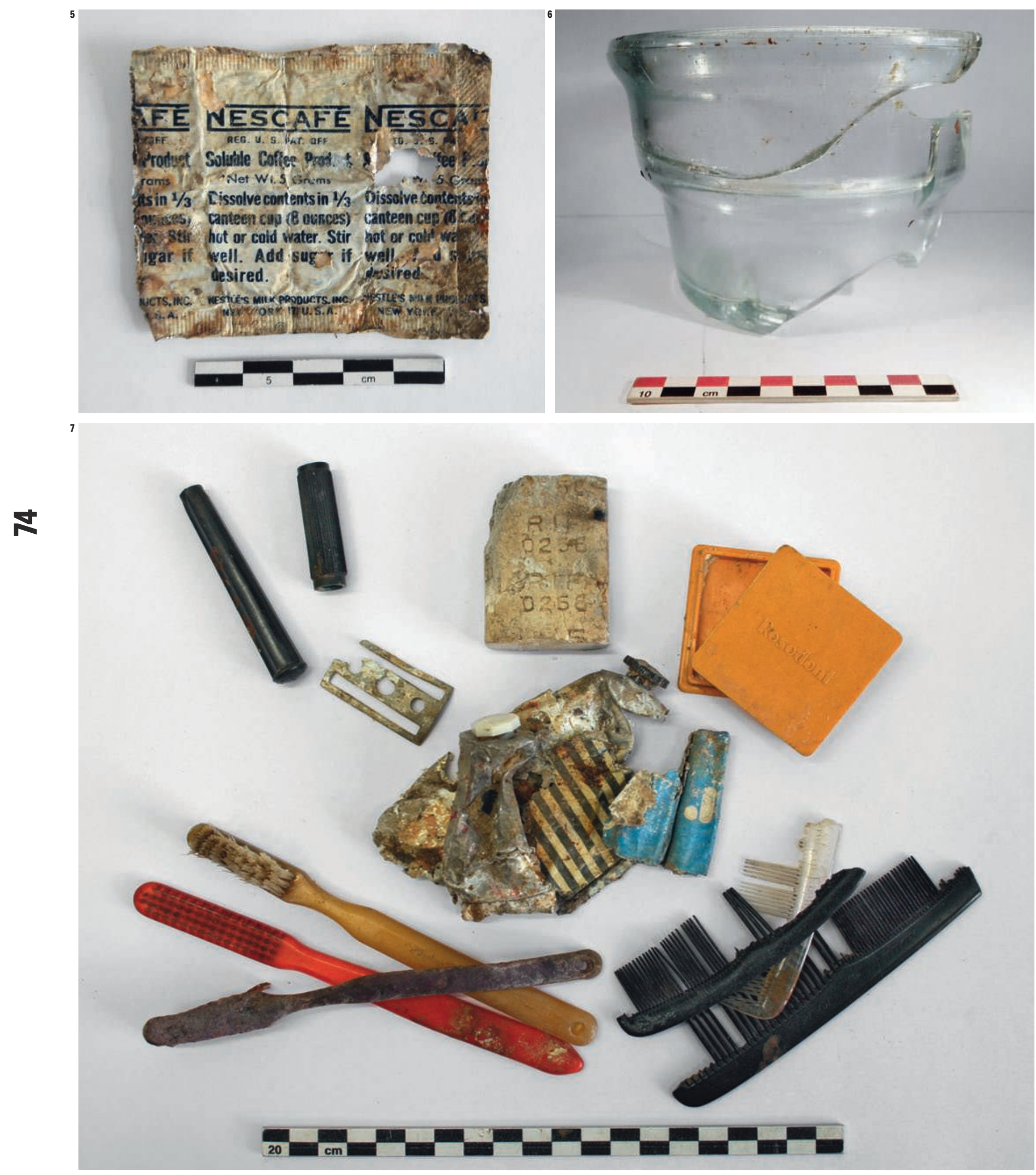

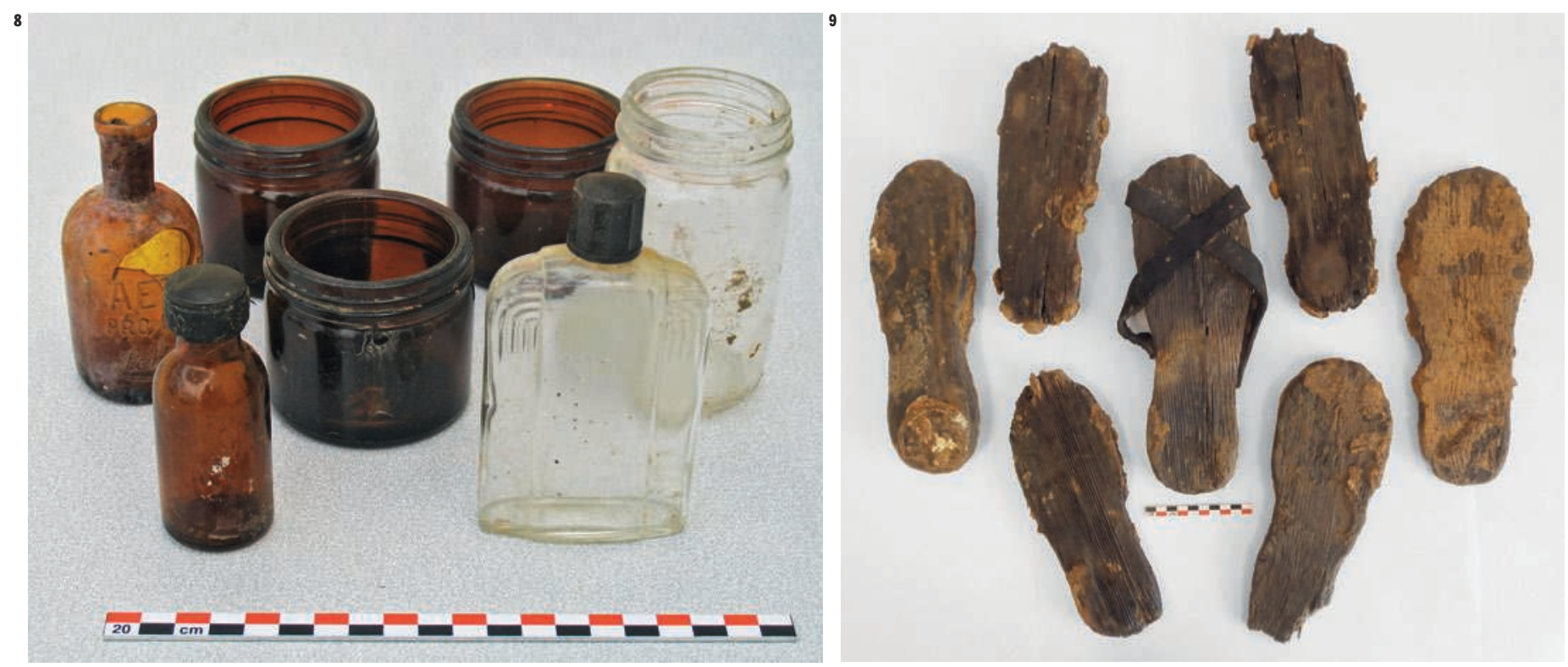

\section{L'opération} archéologique des " Groseilliers ॥ a mis au jour de nombreux objets liés à l'alimentation provenant de l'armée américaine, comme ce sachet de café lyophilisé. 6. Ce bocal en verre témoigne du réemploi dans le camp d'objets à vocation militaire, ici un bocal de mine allemand de type Glasmine 43.

7. Divers effets destinés à l'hygiène corporelle ont été retrouvés. On distingue de gauche à droite et de haut en bas : des manches de rasoir, une lame de rasoir, un savon RIF 0256 d'origine allemande, une boîte en plastique de dentifrice allemand de marque Rosodont, des tubes de dentifrice et de

mousse à raser,

des brosses à dents

américaines, des peignes.

Ce mobilier représente

une faible partie de

celui découvert lors

de l'opération

archéologique de Stenay

« Les Groseilliers v

(Vermard, 2013).

8. Ce lot de flacons et pots

à onguents médicamenteux

découverts lors de

l'opération archéologique

de Stenay « Les

Groseilliers ॥) (Vermard,

2013) témoigne

des préoccupations

sanitaires dans le camp.

9. Ces vestiges de

chaussures fabriquées

à l'aide de matériaux

de récupération montrent

le dénuement des

prisonniers.

\section{Références bibliographiques}

DufilHo A., 2002, Mon lieutenant, un blessé vous demande : la 35e division d'infanterie dans la guerre de 1939-194o, Bordeaux, Toulouse, Les Dossiers d'Aquitaine, Coll. Mémoires de France, 223 p.

FUSSHÖLLER H., 1998, Erinnern ohne Groll: Zweiter Weltkrieg und Gefangenschaft, traduction de VerdysPiel Maryvonne, éd. H. Fusshöller, s.l., 224 p. bastas. pagesperso-orange.fr/pga/temoins/fusshollch3.htm

KAISER H., 1945-1946, Tagebuch von Horst Kaiser, Meine letzten Kriegstage und die Gefangenschaft, http:// www.horst-kaiser.de/Tagebuch.htm

MUSSBACHER F. J., 1945, « Kriegsgefangenschaft» [texte téléchargé en 2007 à l'adresse http://www.8ung.at/ abtnorbert/gefangen.htm, qui ne fonctionne plus aujourd'hui]

www.ajpn.org: http://www.ajpn.org/communeStenay-55502.html, site internet de l'association "Anonymes, Justes et Persécutés durant la période nazie dans les communes de France .
Preventive medicine in world war II, 1969, Medical department, united states army, preventive medicine in world war II, vol. IX, Special Fields, HEATON L. D. ANDERson R. S., Hoff E. C., HofF P. M., Washington, Office of the Surgeon General, Department of the Army, $651 \mathrm{p}$

THÉOFILAKIS F., 2010, Les prisonniers de guerre allemands en mains françaises (1944-1949): captivité en France, rapatriement en Allemagne, Thèse de Doctorat d'Histoire, sous la direction d'Annette Becker, Henry Rousso et Andreas Wirching, Université de Paris-Ouest Nanterre - La Défense, 1653 p. http://bdr.u-paris1o.fr/ theses/internet/2010PA100184_diff.pdf

ThÉOfILAKIS F., 2014, Les prisonniers de guerre allemands. France, 1944-1949, Paris, Fayard, 8oo p.

Vermard L., 2007, Stenay (Meuse), «Aux Cailloux », rapport d'opération, Inrap-SRA Lorraine, $99 \mathrm{p}$

Vermard L. (dir.), AdAM F., Braguier S., Ollive V., 2013 Stenay (Meuse) « Les Groseilliers », rapport d'opération, Inrap-SRA Lorraine, $177 \mathrm{p}$. 